$01 ; 04 ; 12$

\title{
Генерация сгустков электронов лазерным импульсом ультрарелятивистской интенсивности, пересекающим границу неоднородной плазмы
}

\author{
(C) С.В. Кузнецов
}

Объединенный институт высоких температур РАН, Москва, Россия

E-mail: svk-IVTAN@yandex.ru

Поступило в Редакцию 27 февраля 2019 г.

В окончательной редакции 9 апреля 2019 г.

Принято к публикации 9 апреля 2019 г.

Исследован механизм генерации сгустков электронов при взаимодействии лазерного импульса с полуограниченной плазмой, имеющей размытую границу с вакуумом в виде переходного слоя.

Ключевые слова: лазерный импульс, кильватерная волна, полуограниченная плазма, самоинжекция электронов, ускорение электронов.

DOI: 10.21883/PJTF.2019.13.47959.17758

Важной проблемой на пути практической реализации лазерно-плазменного ускорителя, обеспечивающего моноэнергетическое ускорение электронных сгустков, является генерация коротких электронных сгустков, предназначенных для ускорения [1]. Электронные сгустки субфемтосекундной длительности могут быть также использованы для генерации субфемтосекундных рентгеновских импульсов и исследования динамики вещества в межатомном масштабе времени [2-4].

Интересное явление генерации электронных сгустков аттосекундной длительности был обнаружено в работе [5] при численном моделировании процесса распространения мощного лазерного импульса в неоднородной плазме. Считалось, что лазерный импульс падает нормально на размытую границу плазмы, представляющую собой переходный слой, в котором плотность плазмы изменяется по линейному закону с выходом на плато. Оказалось, что при превышении амплитудой лазерного импульса некоторого порогового значения в окрестности точки перехода плотности плазмы на плато развивается квазиодномерный процесс генерации сгустков электронов. Теоретический анализ данного явления в рамках одномерной геометрии был проведен в работах [6-8] на основе простой физической модели, в которой электроны холодной разреженной плазмы рассматривались как плазменные осцилляторы, одномерное движение которых возбуждалось взаимодействующим с ними лазерным импульсом. Однако при этом для упрощения анализа использовалось приближение, согласно которому граница раздела плазмы с вакуумом является резкой. Цель настоящей работы заключается в обобщении теории [6-8] на случай наличия на границе плазмы линейного переходного слоя плотности $n_{0}(z)$ с выходом на плато, как в работе [5].

В работах [6-8] было установлено, что в случае резкой границы плазмы процесс генерации сгустка электронов возможен, если энергия осцилляции
$E_{o s}$, приобретенная электроном от взаимодействия с лазерным импульсом, превышает пороговое значение $E_{o s, t h}=m c^{2} \gamma_{p h}=m c^{2} / \sqrt{1-V_{g r}^{2} / c^{2}}$, где $V_{g r}$ - групповая скорость импульса. При этом процесс генерации электронного сгустка начинается с того электрона, который первоначально находится от границы плазмы на расстоянии, равном амплитуде своих осцилляций $A_{m}=\sqrt{\left(E_{o s}-m c^{2}\right) /\left(2 \pi e^{2} n_{0}\right)}$, где $n_{0}$ - плотность плазмы, $-|e|, m$ - заряд и масса электрона. Поэтому электроны, составляющие сгусток, можно разделить на две группы: выходящие в вакуум в процессе своих осцилляций и не выходящие. Очевидно, что при обобщении задачи на случай нерезкой границы следует ввести изменение, состоящее в том, что теперь электроны будут выходить в переходный слой у границы плазмы. В остальном методология решения задачи остается той же, что в случае резкой границы в работе [8], которой и будем следовать далее.

Пусть на границу плазмы нормально к ее поверхности вдоль оси $O Z$ падает лазерный импульс, несущая частота которого много больше плазменной. Нулевая точка оси $O Z$ располагается в начале плато плотности плазмы. В своем осциллирующем движении электрон может выходить в область $z<0$, поэтому сила, возвращающая его в положение равновесия $z_{0}$, имеет более сложный вид, чем в работе [8], и зависит от плотности неподвижных ионов в переходном слое. Тем не менее и в этом случае можно в квадратурах записать интеграл уравнения движения плазменного осциллятора

$$
\sqrt{m^{2} c^{4}+P^{2} c^{2}}+4 \pi e^{2} \int_{z_{0}}^{z} d z^{\prime} \int_{z_{0}}^{z^{\prime}} n_{0}\left(z^{\prime \prime}\right) d z^{\prime \prime}=E_{o s},
$$

где $P$ - импульс электрона, $z_{0}-$ его центр осцилляции.

В дальнейшем будем предполагать в согласии с результатами численного моделирования, что электроны, 

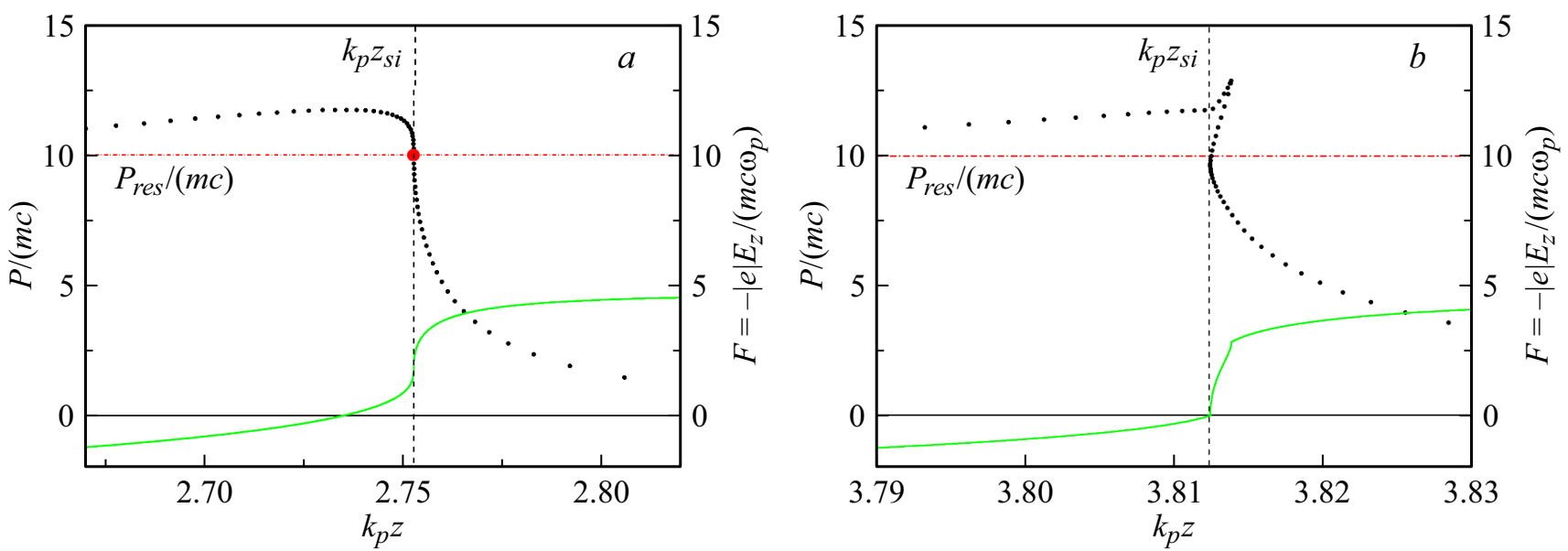

Рис. 1. Распределение на фазовой плоскости $(z, P)$ электронов (точки) и зависимость от координаты $z$ силы $F=-|e| E_{z} /\left(m c \omega_{p}\right)$, действующей на электроны (сплошная линия). $a-$ в момент начала генерации сгустка, $b-$ в момент прекращения процесса аккумулирования в сгусток электронов, располагающихся перед ним.

образующие генерируемый лазерным импульсом сгусток, изначально до воздействия лазерного импульса располагаются на плато плотности плазмы. Тогда сообразно интегралу (1) можно записать для совокупности траекторий таких электронов плазмы общее выражение вида

$$
c t-\frac{c z_{0}}{V_{g r}}=I\left(z, z_{0}, z_{0}\right)+c T_{l f t}\left(z_{0}\right),
$$

где обозначено

$$
\begin{aligned}
& I\left(z_{1}, z_{2}, z_{0}\right)= \\
& =\int_{z_{2}}^{z_{1}} d z^{\prime} / \sqrt{1-m^{2} c^{4} /\left[E_{o s}-2 \pi e^{2} n_{0}\left(z^{\prime}-z_{0}\right)^{2}\right]^{2}} .
\end{aligned}
$$

По форме уравнение (2) идентично, полученному в [8], но функция $T_{l f t}\left(z_{0}\right)$ имеет более сложный вид

$$
\begin{aligned}
& T_{l f t}\left(z_{0}\right)=T_{h}+2\left\{\int_{z_{b n}\left(z_{0}\right)}^{0} d z^{\prime} /\right. \\
& \sqrt{1-m^{2} c^{4} /\left[E_{o s}-4 \pi e^{2} \int_{z_{0}}^{z^{\prime}} d z^{\prime \prime} \int_{z_{0}}^{z^{\prime \prime}} n_{0}\left(z^{\prime \prime \prime}\right) d z^{\prime \prime \prime}\right]^{2}} \\
& \left.-I\left(0, z_{0}-A_{m}, z_{0}\right)\right\} .
\end{aligned}
$$

Запись (2) представляет в области $z>0$ траектории всех электронов плазмы как свободных осцилляторов в тот промежуток времени, когда электроны вновь движутся в направлении распространения лазерного импульса после взаимодействия с ним. Константа интегрирования в формуле (2) определена из условия, что тот электрон, у которого центр осцилляции совпадает с началом координат $z_{0}=0$, в момент времени $t=0$ находится в своем центре осцилляций и движется влево, т. е. выходит в переходный слой. Другие электроны плазмы начинают свое движение по подобной траектории (до выхода в переходный слой) с запаздыванием $z_{0} / V_{g r}$. Приняты также следующие обозначения: $T_{l f t}\left(z_{0}\right)-$ длительность интервала времени, необходимого электрону, чтобы совершить движение из своего центра осцилляции $z_{0}$ влево до крайнего положения $z_{b n}\left(z_{0}\right)$ согласно (1) и вернуться снова в $z_{0}, T_{h}=T_{l f t}\left(A_{m}\right)$ для электронов с центром осцилляции $z_{0}>A_{m}$, не выходящих в переходный слой.

Согласно [6-8], процесс генерации электронного сгустка начинается с пересечения траекторий двух соседних электронов, т.е. он возможен лишь при выполнении условия $d z / d z_{0}=0$. Отсюда, анализируя соотношение (2) для траекторий электронов с учетом их возможного выхода в переходный слой плазмы, получаем результат, аналогичный случаю резкой границы плазмы [8], а именно пороговое значение энергии осцилляции электрона, при котором возможен процесс генерации сгустков, соответствует значению $E_{o s, t h}=m c^{2} \gamma_{p h}=m c^{2} / \sqrt{1-V_{g r}^{2} / c^{2}}$.

$\mathrm{C}$ целью прояснения механизма генерации электронных сгустков было выполнено численное моделирование этого процесса при прохождении циркулярно поляризованного лазерного импульса с огибающей векторного потенциала вида $a=a_{0} \cos ^{2}[t / \tau] \operatorname{sgn}(\pi \tau / 2-|t|)$, где $a_{0}=|e| A_{0} / m c^{2}=7.4-$ безразмерная амплитуда векторного потенциала, $\tau$ - длительность лазерного импульса, соответствующая его длительности на половине высоты $\tau_{\mathrm{FWHM}}=1.143 \tau=12 \mathrm{fs}$ через границу неоднородной плазмы при условии $E_{o s}=11.7437 m c^{2}>E_{o s, t h}$ для аналогичного приведенному в работе [5] профиля ионной плотности в переходном слое с характерной шириной $L_{c h a r}=25 \mu \mathrm{m}$. Групповая скорость $V_{g r}$ распро- 

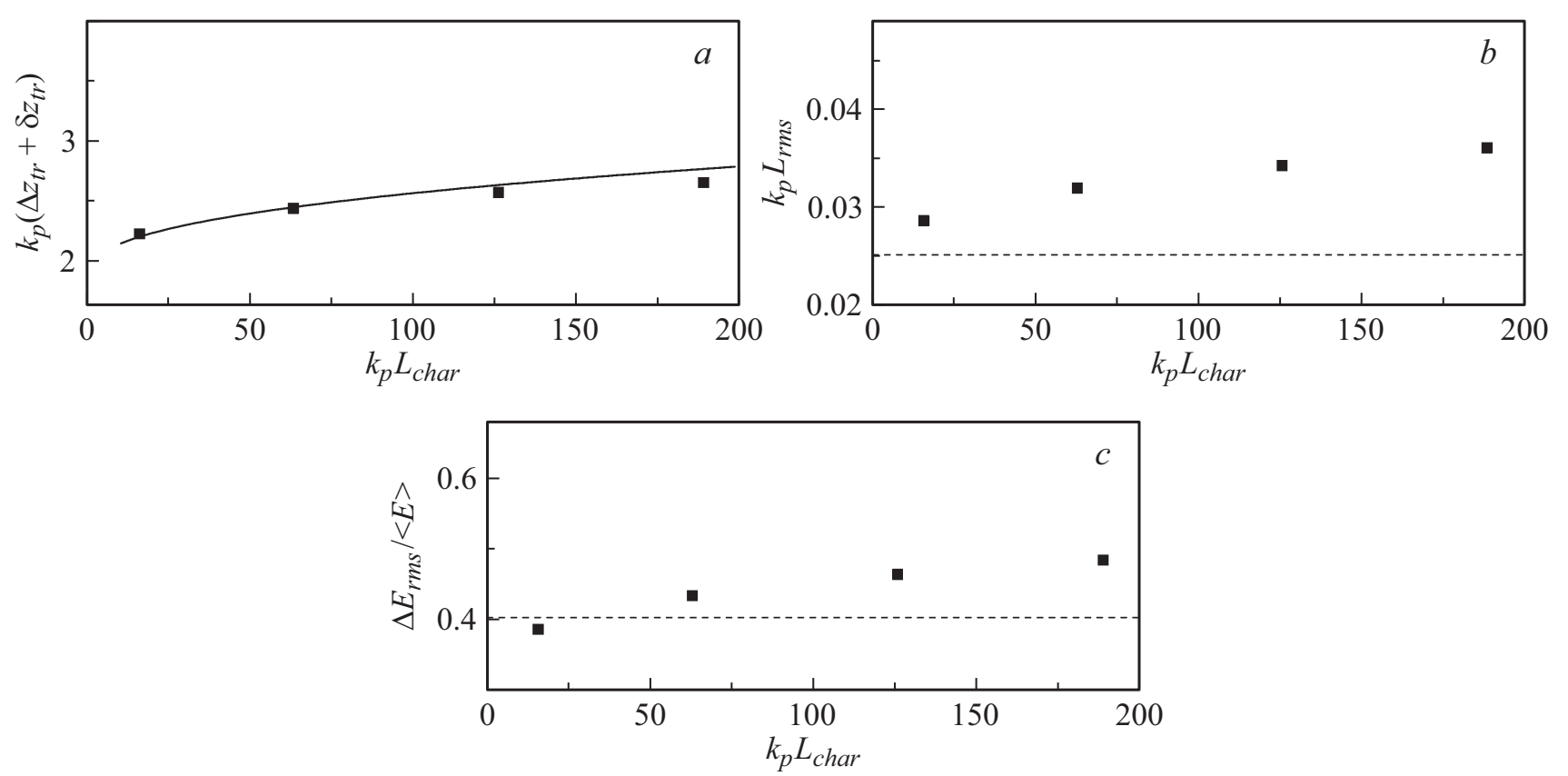

Рис. 2. Толщина слоя $\Delta z_{t r}+\delta z_{t r}$ захватываемых в сгусток электронов $(a)$, длина сгустка $L_{r m s}(b)$ и разброс по энергии в нем $\Delta E_{r m s} /\langle E\rangle(c)$ в зависимости от толщины переходного слоя. Точки - моделирование, сплошная линия - теория с учетом переходного слоя, штриховая линия - аналитическая оценка для случая резкой границы.

странения лазерного импульса при длине его волны $\lambda_{0}=1 \mu \mathrm{m}$ на плато плотности плазмы соответствует гамма-фактору $\gamma_{p h}=1 / \sqrt{1-V_{g r}^{2} / c^{2}}=10$.

Рис. $1, a$ и $b$ последовательно демонстрируют основные моменты процесса генерации сгустка электронов лазерным импульсом, который, как было выяснено в работе [8], по своей физической природе является самоинжекцией электронов в кильватерную волну лазерного импульса с последующим захватом в ней. Рис. 1, $a$ на фазовой плоскости $(z, P)$ показывает момент начала процесса самоинжекции электронов и опрокидывания кильватерной волны. Штриховая линия на нем указывает на оси $O Z$ точку самоинжекции первого электрона $z_{s i}=z_{i n j, f}$, помеченного крупным кружком и имеющего центр осцилляции $z_{0, f}$. Анализ уравнения (2) при условии $d z / d z_{0}=0$ показывает, что величина ускоряющего поля кильватерной волны в этой точке с большой точностью равна $E_{z} \cong-\sqrt{8 \pi n_{0}\left(E_{o s}-\gamma_{p h} m c^{2}\right)}$, а импульс инжектированного электрона $P_{i n j, f} \cong P_{\text {res }}=m c \sqrt{\gamma_{p h}^{2}-1}$. Перемещение лазерного импульса в глубь плазмы и смещение точки самоинжекции $z_{s i}$ электронов вслед за ним приводят к последовательному захвату в кильватерную волну электронов, расположенных дальше от границы плазмы. Одновременно электроны, находящиеся позади первого инжектированного электрона, также последовательно догоняют точку самоинжекции $z_{s i}$ и аккумулируются в сгустке. Когда заряд сгустка достигает такой величины, что он гасит ускоряющее поле на хвосте сгустка, лежащие перед сгустком электроны прекращают захва- тываться в кильватерную волну (рис. $1, b$ ). К этому моменту толщина слоя электронов исходной невозмущенной плазмы, захваченных в кильватерную волну, составляет $\Delta z_{t r} \cong \sqrt{\left(E_{o s}-\gamma_{p h} m c^{2}\right) /\left(4 \pi n_{0}\right)}$. При этом из соотношения (2) определяется значение координаты центра осцилляции догоняющего сгусток электрона $z_{0, q}$, который последним на данный момент аккумулируется в нем:

$$
\begin{aligned}
T_{l f t}\left(z_{0, q}\right)= & \frac{\sqrt{\left(E_{o s}-\gamma_{p h} m c^{2}\right) /\left(2 \pi e^{2} n_{0}\right)}}{V_{g r}} \\
& +\frac{1}{c} I\left(z_{i n j, f}, z_{0, f}, z_{0, f}\right)+T_{h} .
\end{aligned}
$$

Далее в сгусток аккумулируются только догоняющие электроны. Но поскольку при своем отклонении от центров осцилляции их скорости снижаются, в некоторый момент времени их аккумуляция в сгусток прекращается тоже. Последний электрон из догоняющих, который достигает бегущей точки $z_{s i}$, имеет центр осцилляции в точке $z_{0, l}$, которая, согласно (2), удовлетворяет соотношению (см. также [8])

$$
\begin{aligned}
T_{l f t}\left(z_{0, l}\right)= & \frac{2 \sqrt{\left(E_{o s}-\gamma_{p h} m c^{2}\right) /\left(2 \pi e^{2} n_{0}\right)}}{V_{g r}} \\
& +\frac{2}{c} I\left(z_{i n j, f}, z_{0, f}, z_{0, f}\right)+T_{h} .
\end{aligned}
$$

Используя разложение $T_{l f t}\left(z_{0}\right)$ по степеням $\varepsilon=A_{m}-z_{0} \ll A_{m} \quad$ при условии $\left|z_{b n}\left(z_{0}\right)\right| \ll L_{c h a r}$, 


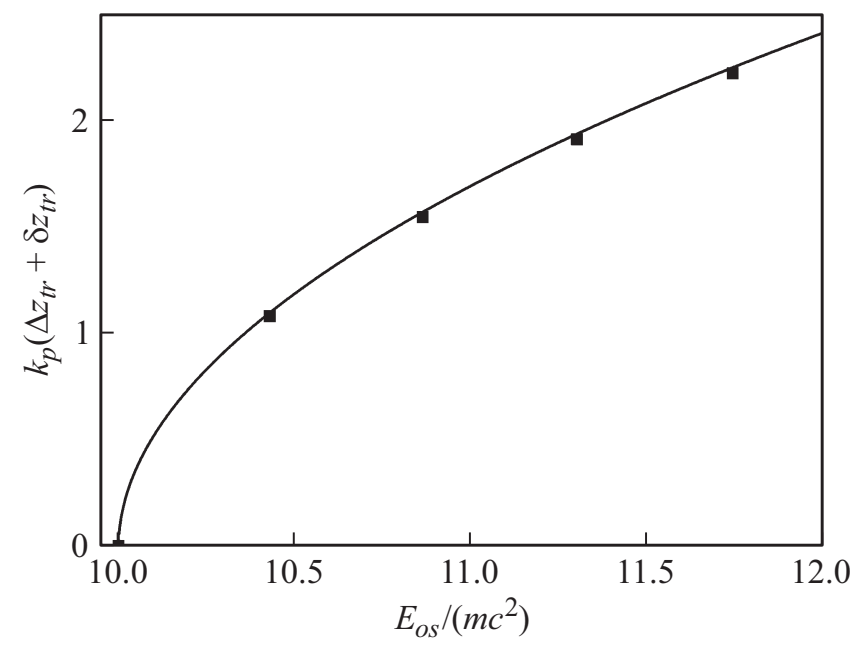

Рис. 3. Толщина слоя $\Delta z_{t r}+\delta z_{t r}$ захватываемых в сгусток электронов плазмы в зависимости от энергии плазменных осцилляторов, возбуждаемых лазерным импульсом. Сплошная линия и точки - соответственно аналитический результат и моделирование для плазмы с переходным слоем $L_{\text {char }}=25 \mu \mathrm{m}$.

можно решить совместно уравнения (3), (4) и найти формулу

$$
\begin{aligned}
& k_{p} \delta z_{t r}=\left(\frac{15 k_{p} L_{c h a r}}{4 \sqrt{2}}\right)^{2 / 5}\left(2^{2 / 5}-1\right)\left[2\left(\frac{E_{o s}}{m c^{2}}-1\right)\right]^{3 / 5} \\
& \times\left[2\left(\frac{E_{o s}}{m c^{2}}-\gamma_{p h}\right)\right]^{1 / 5}\left[\frac{1}{\sqrt{1-1 / \gamma_{p h}^{2}}}-\frac{1}{\sqrt{1-m^{2} c^{4} / E_{o s}^{2}}}\right]^{2 / 5}
\end{aligned}
$$

для толщины добавочного слоя электронов плазмы $\delta z_{t r}=z_{0, q}-z_{0, l}$, накапливаемых в генерируемом лазерным импульсом сгустке, где обозначено $k_{p}=\sqrt{4 \pi e^{2} n_{0} / m c^{2}}$.

Рис. 2, $a$ показывает зависимость толщины слоя аккумулируемых в сгустке электронов плазмы от размера переходной области в типичном диапазоне ее изменения $L_{\text {char }}=20-300 \mu \mathrm{m}$ и демонстрирует близость расчетов по формулам к результатам моделирования. В этом же диапазоне на рис. 2, $b$ и $c$ по результатам моделирования (точки) показаны асимптотические значения длины сгустка и разброса электронов по энергии в нем на больших длинах ускорения сгустка электронов. Эти характеристики с достаточной точностью могут быть оценены по аналитическим формулам работы [7] для случая резкой границы (штриховая линия).

Зависимость от энергии плазменных осцилляторов толщины слоя электронов плазмы, собираемых в сгустке, при значении $L_{c h a r}=25 \mu \mathrm{m}$ показана на рис. 3. Хорошее согласие аналитических результатов с численным моделированием подтверждает адекватность используемой физической модели для анализа явления генерации сгустка электронов при наличии в плазме переходного слоя.

\section{Список литературы}

[1] Костюков И.Ю., Пухов А.М. // УФН. 2015. Т. 185. № 1. C. 89-96.

[2] Sell A., Kärtner F.X. // J. Phys. B. 2014. V. 47. P. 015601.

[3] Lewenstein M. // Science. 2002. V. 297. P. 1131-1132.

[4] Corkum P.B., Krausz F. // Nature Phys. 2007. V. 3. P. 381-387.

[5] Li F.Y., Sheng Z.M., Liu Y., Meyer-ter-Vehn J., Mori W.B., Lu W., Zhang J. // Phys. Rev. Lett. 2013. V. 110. N 13. P. 135002.

[6] Кузнецов С.В. // Письма в ЖТФ. 2016. Т. 42. В. 14. С. 52-58.

[7] Кузнецов С.В. // ЖЭТФ. 2016. Т. 150. В. 2 (8). С. 195-213.

[8] Кузнецов С.В. // Квантовая электроника. 2018. Т. 48. № 10. C. $945-953$. 